\title{
Identification of IncRNA competitively regulated subpathways in myocardial infarction
}

\author{
$\mathrm{XIA} \mathrm{WU}^{1 *}$, LILI SUN $^{1 *}$ and ZILIANG WANG ${ }^{2}$ \\ ${ }^{1}$ Department of Geriatrics, Daqing Oilfield General Hospital; ${ }^{2}$ Department of Cardiovascular Medicine, \\ Daqing People's Hospital, Daqing, Heilongjiang 163316, P.R. China
}

Received June 26, 2018; Accepted February 6, 2019

DOI: $10.3892 /$ etm.2019.8210

\begin{abstract}
The functions of long non-coding RNAs (lncRNAs) in myocardial infarction (MI) remain largely unknown. Thus, we used the subp athway-LINCE method to characterize the potential roles of lncRNAs in MI. Candidate lncRNA-mRNA interactions were obtained from miRNA-mRNA interactions and IncRNA-miRNA interactions. Then the lncRNA and mRNA co-expression relationship pairs (LncGenePairs) were screened from the lncRNAs and mRNA intersections, which were extracted through candidate lncRNA-mRNA interactions and sample gene expression profiles. The lncRNAs in LncGenePairs were embedded into pathway graphs as nodes through linking to their regulated mRNAs, which resulted in obtaining condition-specific lncRNA competitively regulated signal pathways (csLncRPs). Finally, the csLncRPs were calculated using lenient distance similarity to obtain the lncRNA competitively regulated subpathways. Based on the statistical significance of signal subpathways, IncRNA-mRNA networks were constructed, in which hub IncRNAs were selected. A total of 65 lncRNAs competitively regulated subpathways and 13 hub lncRNAs were obtained, which associated with a risk of MI. Identifying lncRNAs competitively regulated subpathways not only provides potential lncRNA biomarkers for MI, but also helps the understanding of pathogenesis of MI.
\end{abstract}

\section{Introduction}

Myocardial infarction (MI), a common presentation for ischemic heart disease/coronary artery disease, is a major contributor to mortality rates worldwide (1). The major trigger of MI is generally owing to thrombus formation in a coronary

Correspondence to: Dr Ziliang Wang, Department of Cardiovascular Medicine, Daqing People's Hospital, 241 Jianshe Road, High New Technology Research and Development Zone, Daqing, Heilongjiang 163316, P.R. China

E-mail: wangziliangdaqing@163.com

${ }^{*}$ Contributed equally

Key words: IncRNA, mRNA, subpathway, network, biomarker, myocardial infarction artery. Accordingly, the current treatments mainly include medical therapy (e.g. anticoagulant medications, antiplatelets) and elective catheterization. However, some disadvantages can not be ignored, such as high risk of bleeding in medical therapy and high cost of treatment in elective catheterization (2). In order to effectively prevent and treat MI, seeking the exact pathogenesis of MI at various aspects is necessary. Currently research directions mainly include improvement of coronary blood flow, inhibition of apoptosis, reduction of oxygen consumption, and revascularization procedures (3). However, few studies have been conducted in the pathogenesis of MI at the non-coding RNA (ncRNA) level.

ncRNAs, RNA molecules, are not translated into proteins, which mainly include ribosomal RNAs (rRNAs), transfer RNAs (tRNAs), short non-coding RNAs such as microRNAs (miRNAs) and the long non-coding RNAs (lncRNAs). lncRNAs are a set of RNAs longer than 200 nucleotides, and participate in many fundamental biological processes mainly including genomic imprinting and chromatin modification (4). Although the effects of lncRNAs on various cancers (5) and neuronal diseases $(6,7)$ have been widely investigated, the study number of lncRNA function in cardiovascular diseases is very limited. Currently, some transcripts have been investigated for their potential role as biomarkers of cardiovascular diseases. The mitochondrial IncRNA LIPCAR, as a novel biomarker, can predict future death in heart failure patients (8). IncRNA CoroMarker is a diagnostic biomarker for coronary artery disease (9). In addition, lncRNA MIAT might regulate MI via functioning as a competing endogenous RNA for various targets $(10,11)$.

miRNAs are short non-coding RNAs (approximately 20 nucleotides) and negatively regulate target genes. Different from lncRNAs, miRNAs have been largely investigated in the context of MI (12). The relationship between miRNAs and IncRNAs has been extensively demonstrated, among which IncRNAs can indirectly compete with mRNAs through binding to miRNAs $(13,14)$. For instance, lncRNA CHRF could regulate cardiac hypertrophy by targeting miR-489 (15). Recognizing lncRNA competitively regulated subpathway can reveal the pathogenesis of disease and the molecular mechanism of lncRNAs in the disease context. However, the relevant regulated mechanism of lncRNAs in MI remains unclear. Subpathway-LNCE, as a novel method, can effectively integrate lncRNA-mRNA expression profile and identify lncRNA competitively regulated subpathway (16). Moreover, 
subpathway-LNCE method is more accurate, advanced and its calculation results are more relevant to disease. Accordingly, we used the subpathway-LNCE method to investigate pathogenesis of MI at genetic level.

\section{Materials and methods}

Recruitment and pretreatment of gene expression data. Gene expression profiles with accession number (GSE34198) for MI were recruited from the Gene Expression Omnibus (GEO) (http://www.ncbi.nlm.nih.gov/geo/) database. GEO is a public functional genomics data repository that freely distributes high throughput gene expression profiles and other functional genomics data sets. GSE34198 consists of 48 healthy controls and 49 patients with MI, and is deposited on GPL6102 data platform.

For purpose of controlling the quality of GSE34198, GSE34198 was pretreated as follows: Background correction was performed by Robust Multi-array Average (RMA) algorithm (17), the normalization was evaluated by quantiles algorithm (18), perfect match and mismatch was revised by Micro Array Suite (MAS) algorithm (19), and all expression values were summarized by medianpolish method (17). After removing invalid or duplicated probes, we converted them into gene symbols by the annotate package (20). Thus, 19,027 genes were obtained in the pretreated GSE34198 of MI for further application.

Constructing the candidate lncRNA-mRNA interactions. As far as we known, small non-coding RNAs target Base version 2.0 (starBase v2.0, http://starbase.sysu.edu.cn/) has been used to identify the RNA-RNA and protein-RNA interaction networks. Hence, miRNA-lncRNA interactions and miRNA-mRNA interactions were collected using StarBase v2.0. The miRNA-mRNA interactions were acquired based on miRecords (21), mir2Disease (22), mirTarBase (23) and TarBase (24). Then candidate lncRNA-mRNA competitively regulated relationships were constructed using their shared miRNAs between miRNA-mRNA and miRNA-lncRNA interactions. To ensure the data reliability, we used two criteria to identify the candidate competing miRNA of each lncRNA as follows: i) hypergeometric test of shared miRNAs under a threshold of $\mathrm{P}=0.05$ and ii) Jaccard Coefficient of shared miRNAs rank at top $20 \%$.

To make the candidate lncRNA-mRNA interactions involved in MI, all the genes in GSE34198 were mapped on them and the intersections were selected for further analysis.

Obtaining the IncRNA and mRNA co-expression relationship pairs. To screen the candidate lncRNA-mRNA interactions, we evaluated co-expression for any pair of relations in the candidate lncRNA-mRNA network using Pearson's correlation coefficient. Subsequently, the significance of Pearson's correlation coefficient was evaluated by Fisher's Z-transform, which converts the values into the normally distributed variable $\mathrm{Z}$. Then the Z-transform test utilizes the one-to-one mapping of the standard normal curve to the P-value of a one tailed test. When the calculation value exceeded a significant positive threshold
$(\mathrm{P}<0.05)$, the lncRNA-mRNA co-expression relationship pairs (LncGenePairs) were retained.

Reconstructing condition-specific IncRNA competitively regulated signal pathways. We used Fisher's test to identify the gene enrichment pathways in the mRNA-gene expression profiles, and the pathways were obtained from KEGG database. The gene enrichment pathways were collected when the P-value of gene enrichment pathway was no longer than 0.01 , and considered as the candidate difference pathways. We put IncRNAs in the LncGenePairs into the candidate difference pathway graphs as nodes, then we acquired condition-specific lncRNA regulated signal pathways (csLncRPs). The lncRNA nodes were considered as signature nodes.

Located subpathways within pathways according to signature nodes. Signature nodes represent information on the competing regulation and genes of interest, which can help to efficiently locate subpathways through further considering their topologies within pathways. Moreover, distances are usually similar between certain nodes in a subpathway. We utilized 'lenient distance' similarity of signature nodes to locate subpathways competing for regulation by lncRNAs. We computed the shortest path between any two signature nodes as follows: If the molecule number between two signature nodes was less than $\mathrm{n}$, they were combined into a single node. Finally, the node number in the molecule sets within pathway was longer than s, and defined as subpathway regions. The $\mathrm{n}$ parameter conducts the intensity of regulated signals, and the s parameter regulates the size of candidate subpathways. Thus, $n=1$ and $s=8$ were used as default parameters.

Statistical significance of candidate subpathways. Wallenius approximation methods were applied to evaluate the statistical significance of each subpathway. The needed values were shown as follows: i) the number of interesting mRNAs (x) submitted for analysis; ii) background mRNAs (n) number; iii) the number of background mRNAs (m1) annotated to each subpathway; iv) the number of interesting mRNAs (m2) annotated to each subpathway and v) the weight of each subpathway (w), which indicated the intensity of competitively regulated lncRNAs involved in this subpathway. The formula of the subpathway weight is as follows:

$$
W=1+\beta\left(-\log _{2}\left(\frac{G_{L}}{P_{G}}\right)\right)
$$

According to the formula above, $\mathrm{P}_{\mathrm{G}}$ represents the number of mRNAs in the subpathways. $G_{L}$ represents the number of mRNAs competing regulation by lncRNAs in this subpathway. Moreover, $\beta$ is the control parameter $(\beta=1)$. The Wallenius approximation methods were carried out by $\mathrm{R}$ package BiasedUrn (25).

Identifying hub lncRNAs. According to the values of the subpathways, the subpathways competing for regulation by lncRNAs were obtained and constructed, that is, 
Table I. The diagram of the top 6 LncGenePairs.

\begin{tabular}{llcc}
\hline Lnc & Gene & corValue & P-value \\
\hline TDRG1 & HENMT1 & 0.220560501443871 & 0.0299373311676897 \\
LIMD1-AS1 & LRRC56 & 0.246484637523062 & 0.0149413386406672 \\
LEF1-AS1 & MAGEH1 & 0.454787669314928 & $2.87221540632211 \times 10^{-6}$ \\
SNHG11 & MRPL53 & 0.680510717664986 & $1.75464015432401 \times 10^{-14}$ \\
LINCO0485 & NME7 & 0.249365243302853 & 0.0137715015048636 \\
LEF1-AS1 & SLC27A5 & 0.424727193889112 & 0.0000145102562949063
\end{tabular}

Table II. The diagram of the top 6 candidate different pathways.

\begin{tabular}{llll}
\hline Index & \multicolumn{1}{c}{ Pathway_id } & Pathway_pvalue & p.adjust (FDR) \\
\hline 05215 & hsa05215: Prostate cancer & $9.99732401124532 \times 10^{-12}$ & $9.99732401124532 \times 10^{-12}$ \\
05200 & hsa05200: Pathways in cancer & $8.45066918278111 \times 10^{-14}$ & $8.45066918278111 \times 10^{-14}$ \\
05220 & hsa05220: Chronic myeloid leukemia & $7.81664671803157 \times 10^{-17}$ & $7.81664671803157 \times 10^{-17}$ \\
04910 & hsa04910: Insulin signaling pathway & 0.0000771758111031205 & 0.0000771758111031205 \\
04510 & hsa04510: Focal adhesion & $6.65828057822821 \times 10^{-06}$ & $6.65828057822821 \times 10^{-06}$ \\
05166 & hsa05166: HTLV-I infection & $6.59501272205171 \times 10^{-07}$ & $6.59501272205171 \times 10^{-07}$ \\
\hline
\end{tabular}

IncRNA-mRNA networks were constructed. Then, we collected the hub lncRNAs when the lncRNA degree was longer than the average lncRNA degree in the lncRNA-mRNA networks.

\section{Results}

Identifying the relationship between IncRNAs and mRNAs for MI. In this study, 19,027 genes were selected from GSE34198 of MI after standard corrections and normalizations. To understand the relationship between IncRNA and mRNA, we extracted IncRNA-mRNA interactions by their shared miRNAs based on mRNA-miRNA interactions and IncRNA-miRNA interactions. All genes in GSE34198 were mapped on these lncRNA-mRNA interactions and the intersections were selected. We then collected these intersections which satisfied $\mathrm{P}<0.05$. The results revealed that 7,693 $\operatorname{lncRNA}-\mathrm{mRNA}$ interactions were obtained, which included 835 lncRNAs and 1,749 mRNAs. Next, 1,681 mRNAs and 112 lncRNAs were indentified for MI after taking intersections with 19,027 genes in gene expression data. Subsequently, Pearson's correlation coefficient was performed to evaluate co-expression for any pair of relations in the intersections. We obtained 300 lncRNAmRNA co-expression relationship pairs (LncGenePairs), among which include 58 lncRNAs, and 243 mRNAs. The top 6 LncGenePairs are illustrated in Table I.

Reconstruction of condition-specific lncRNA competitively regulated signal pathways. In order to obtain the lncRNA competitively regulated signal pathways (csLncRPs), we firstly annotated the mRNAs in the mRNAs-gene expression profiles into KEGG pathways. A total of 62 candidate difference pathways were obtained, among which the top 6 pathways were selected as shown in Table II. We then put lncRNAs based
Table III. The diagram of the top 14 csLncRPs.

\begin{tabular}{lll}
\hline path_name & matched_lnc & matched_gene \\
\hline 3008 & DLEU2 & BMS1 \\
3008 & C14orf169 & BMS1 \\
3008 & ERVK13-1 & RCL1 \\
3008 & PCBP1-AS1 & POP1 \\
3008 & ERVK13-1 & MDN1 \\
3008 & ERVK13-1 & WDR75 \\
3008 & ERVK13-1 & UTP15 \\
3008 & PDXDC2P & HEATR1 \\
3008 & ERVK13-1 & WDR43 \\
3008 & ERVK13-1 & LSG1 \\
3010 & ERVK13-1 & RPS6 \\
3010 & ERVK13-1 & RPS27 \\
3010 & ERVK13-1 & RPS25 \\
3010 & ERVK13-1 & RPS3A \\
\hline
\end{tabular}

on the LncGenePairs in the candidate difference pathways as nodes through linking to their regulated mRNAs. Hence, a total of 1,241 csLncRPs were acquired, among which the top 14 pathways were selected as shown in Table III.

Identifying the signal subpathways competitively regulated by lncRNA for MI. The csLncRPs were calculated using lenient distance similarity toobtain thelncRNA competitively regulated subpathways. Then the Wallenius approximation methods were performed to evaluate the significance of signal subpathways. A total of 65 lncRNA competitively regulated subpathways were obtained, among which the top rank 6 subpathways are listed in Table IV. We illustrated the schematic diagram of 
Table IV. The diagram of the top six of the IncRNA competitively regulated subpathways.

\begin{tabular}{llccccc}
\hline Pathway Id & \multicolumn{1}{c}{ Pathway name } & $\begin{array}{c}\text { Molecule } \\
\text { ratio }(\mathrm{m} 2 / \mathrm{x})\end{array}$ & Bg ratio $(\mathrm{m} 1 / \mathrm{n})$ & Weight & P-value & FDR \\
\hline 03010_1 & Ribosome & $13 / 264$ & $13 / 26232$ & 1.000000 & $<0.001$ & $0.0000 \mathrm{E}+0000$ \\
03013_1 & RNA transport & $11 / 264$ & $13 / 26232$ & 1.241008 & $<0.001$ & $0.0000 \mathrm{E}+0000$ \\
$04010 \_1$ & MAPK signaling pathway & $17 / 264$ & $25 / 26232$ & 1.556393 & $<0.001$ & $0.0000 \mathrm{E}+0000$ \\
04012_1 & ErbB signaling pathway & $10 / 264$ & $15 / 26232$ & 1.736966 & $<0.001$ & $0.0000 \mathrm{E}+0000$ \\
04062_1 & Chemokine signaling pathway & $14 / 264$ & $20 / 26232$ & 1.514573 & $<0.001$ & $0.0000 \mathrm{E}+0000$ \\
04066_1 & HIF-1 signaling pathway & $11 / 264$ & $16 / 26232$ & 1.540568 & $<0.001$ & $0.0000 \mathrm{E}+0000$ \\
\hline
\end{tabular}

lncRNAs, long non-coding RNA; MAPK, mitogen activated protein kinase.
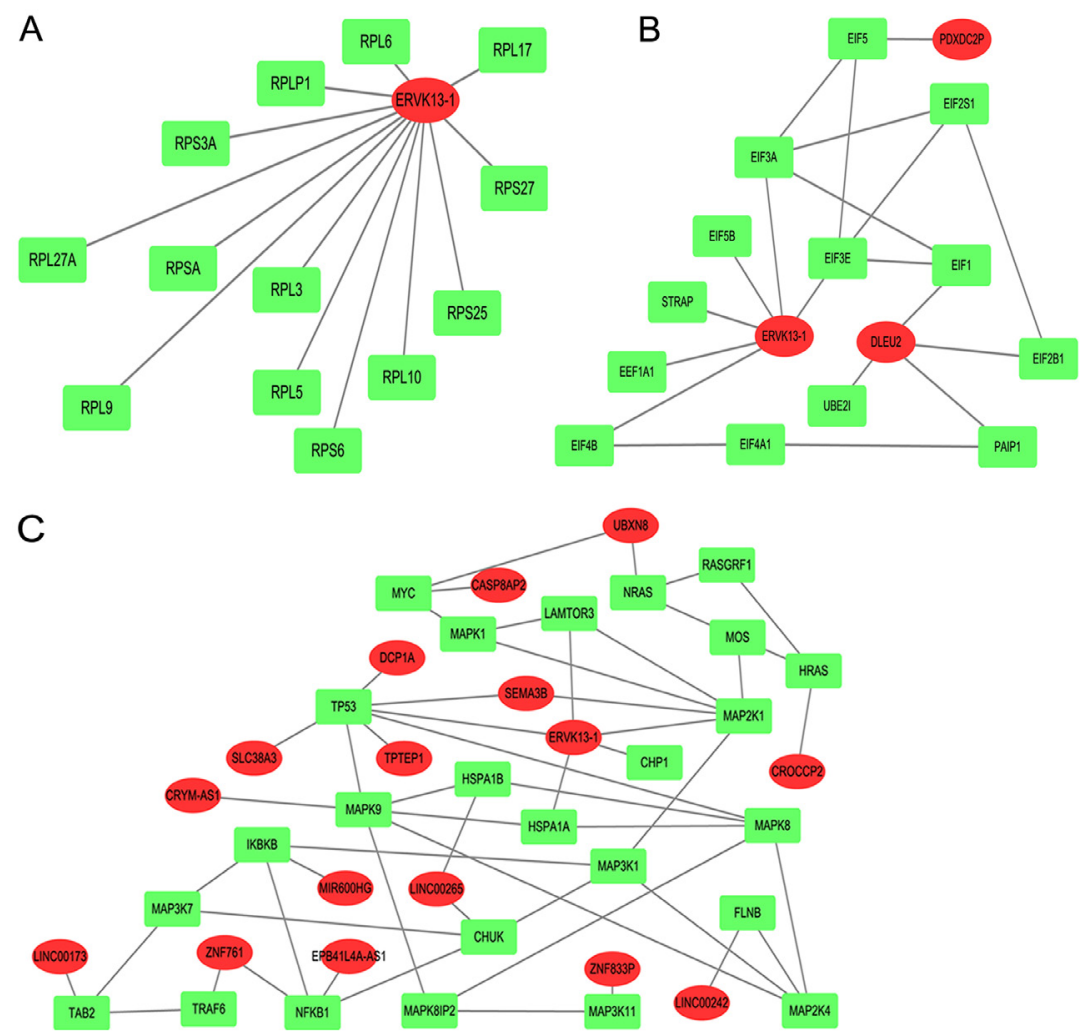

Figure 1. The diagram of the top three subpathways: (A) the ribosome subpathways; (B) the MAPK signaling subpathways; (C) the RNA transport subpathways. Green, mRNA; red, lncRNA. MAPK, mitogen activated protein kinase; lncRNA, long non-coding RNA.

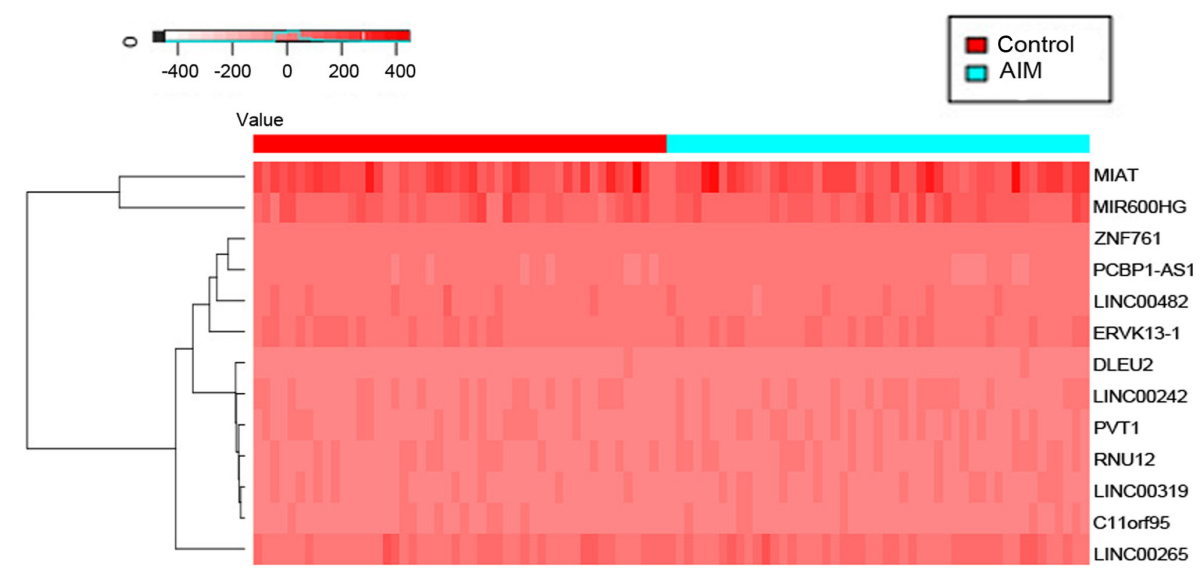

Figure 2. The heatmap indicates the expression quantity of hub lncRNAs (rows) for two groups of patients (colums). The degree of expression is indicated by different colors, with expression increasing between white and red. White, low expression; red, high expression. lncRNA, long noncoding RNA. 
top rank 3 lncRNA competitively regulated subpathways, that is Ribosome, RNA transport and mitogen activated protein kinase (MAPK) subpathways (Fig. 1). According to the value, the signal subpathways were constructed into lncRNA-mRNA networks. Then 13 hub lncRNAs were collected from the lncRNA-mRNA networks (Fig. 2).

\section{Discussion}

In our study, the subpathway-LNCE method was first used to identify the lncRNAs competitively regulated subpathways for MI. Then the IncRNA-mRNA network was constructed according to these signal subpathways, in which the hub lncRNAs were detected.

Plenty of literature has reported that 1 ncRNAs regulate many fundamental biological processes and play a key role in various diseases $(4,5)$. Besides, lncRNAs competitively regulate mRNA expression levels through binding to miRNAs, so that lncRNAs can maintain normal biological functions $(13,14)$. Hence, identifying the functional relationships between lncRNA and disease relevant subpathways may help understand the pathogenesis of diseases. However, the relevant regulated mechanism of 1 ncRNAs in MI remains unclear. Thus, it is very important to search for a suitable method to examine the functions of lncRNAs in MI.

Therefore, we applied subpathway-LINCE with GEO data set of MI and identified a total of 65 lncRNA competitively regulated subpathways under the condition of $\mathrm{P}<0.01$. Of which, P-values of 36 subpathways were nearly zero, which showed the significant difference between MI group and control group, such as Ribosome, RNA transport and MAPK subpathways. Ribosome subpathways are critical to ribosome assembly and protein synthesis. When using some methods to regulate the ribosome biogenesis, the cell growth will be extensively affected. For example, mTOR signaling can regulate multiple steps in ribosome biogenesis, thus influencing the cell proliferation and survival $(26,27)$. Moreover, mTOR is activated in MI and mTOR inhibition could reduce cardiac dilation and infract size and improve cardiac function (28). Hence, clarifying the effect of ribosome subpathways on MI may offer opportunities for new therapies. RNA transport is the process where specific RNA molecules are transported from one cellular region to another via different sorting and transport mechanisms (29). In the present study, RNA transport subpathway in MI was associated with lncRNAs, which might assist in better understanding of the pathogenesis of MI. In particular, MAPK influences mitochondria mediated cell functions, mainly including proliferation, apoptosis and gene expression, because mitochondria are great power providers and gate-keepers of cell life and death. Moreover, MAPK can significantly affect cellular signaling underlying cardiac compensation and decompensation via interacting with the mitochondria (30). Our study showed that MAPK signaling subpathways participate in the pathological process of MI and are involved in lncRNAs. This could help to clarify the pathogenesis of MI.

In order to explore hub lncRNAs in the signal subpathways, the lncRNA-mRNA network was constructed through selecting the high degree of the subpathways. Then, a total of 13 hub lncRNAs were collected from the lncRNA-mRNA networks. Among these hub lncRNAs, MI associated transcript (MIAT), as an lncRNA, has been associated with a risk of MI $(10,31)$. It has been reported that MIAT expression levels are found to change in peripheral blood cells in patients who have suffered from MI, and smoking as a cardiovascular risk factor is found to be positively associated with MIAT (32). Apart from MIAT, other new hub lncRNAs for MI were found using subpathway-LINCE in this study. These hub lncRNAs could become potential diagnostic and therapeutic targets for MI.

In conclusion, using subpathway-LINCE to study MI lncRNA competitively regulated subpathways were gained, and the hub lncRNAs for MI in lncRNA-mRNA network were also obtained. Identifying the lncRNAs competitively regulated subpathways could help us to understand the pathogenesis of MI. The hub lncRNAs might represent novel regulators of MI and become new diagnostic and therapeutic targets for MI. Although the results in this study still need to be verified by experiments, these findings can help understand the roles of lncRNAs in MI.

\section{Acknowledgements}

Not applicable.

\section{Funding}

No funding was received.

\section{Availability of data and materials}

The datasets used and/or analyzed during the present study are available from the corresponding author on reasonable request.

\section{Authors' contributions}

XW and LS conceived the study and drafted the manuscript. XW acquired the data. LS and ZW analyzed the data and revised the manuscript. All authors read and approved the final study.

\section{Ethics approval and consent to participate}

Not applicable.

\section{Patient consent for publication}

Not applicable.

\section{Competing interests}

The authors declare that they have no competing interests.

\section{References}

1. Frangogiannis NG: Pathophysiology of myocardial infarction. Compr Physiol 5: 1841-1875, 2015.

2. Boateng S and Sanborn T: Acute myocardial infarction. Dis Mon 59: 83-96, 2013.

3. Heyn J, Hinske C, Möhnle P, Luchting B, Beiras-Fernandez A and Kreth S: MicroRNAs as potential therapeutic agents in the treatment of myocardial infarction. Curr Vasc Pharmacol 9: 733-740, 2011. 
4. Wang K, Liu CY, Zhou LY, Wang JX, Wang M, Zhao B, Zhao WK, Xu SJ, Fan LH, Zhang XJ, et al: APF lncRNA regulates autophagy and myocardial infarction by targeting miR-188-3p. Nat Commun 6: 6779, 2015.

5. Huarte M: The emerging role of lncRNAs in cancer. Nat Med 21: $1253-1261,2015$

6. Clark BS and Blackshaw S: Long non-coding RNA-dependent transcriptional regulation in neuronal development and disease. Front Genet 5: 164, 2014.

7. Iyengar BR, Choudhary A, Sarangdhar MA, Venkatesh KV, Gadgil CJ and Pillai B: Non-coding RNA interact to regulate neuronal development and function. Front Cell Neurosci 8: 47, 2014.

8. Kumarswamy R, Bauters C, Volkmann I, Maury F, Fetisch J, Holzmann A, Lemesle G, de Groote P, Pinet F and Thum T: Circulating long noncoding RNA, LIPCAR, predicts survival in patients with heart failure. Circ Res 114: 1569-1575, 2014.

9. Yang Y, Cai Y,Wu G, Chen X, Liu Y, Wang X, Yu J, Li C, Chen X, Jose PA, et al: Plasma long non-coding RNA, CoroMarker, a novel biomarker for diagnosis of coronary artery disease. Clin Sci (Lond) 129: 675-685, 2015.

10. Ishii N, Ozaki K, Sato H, Mizuno H, Saito S, Takahashi A, Miyamoto $\mathrm{Y}$, Ikegawa $\mathrm{S}$, Kamatani $\mathrm{N}$, Hori $\mathrm{M}$, et al: Identification of a novel non-coding RNA, MIAT, that confers risk of myocardial infarction. J Hum Genet 51: 1087-1099, 2006.

11. Yan B, Yao J, Liu JY, Li XM, Wang XQ, Li YJ, Tao ZF, Song YC, Chen Q and Jiang Q: IncRNA-MIAT regulates microvascular dysfunction by functioning as a competing endogenous RNA. Circ Res 116: 1143-1156, 2015.

12. Busch A, Eken SM and Maegdefessel L: Prospective and therapeutic screening value of non-coding RNA as biomarkers in cardiovascular disease. Ann Transl Med 4: 236, 2016.

13. Tay Y, Rinn J and Pandolfi PP: The multilayered complexity of ceRNA crosstalk and competition. Nature 505: 344-352, 2014.

14. Yoon JH, Abdelmohsen K and Gorospe M: Functional interactions among microRNAs and long noncoding RNAs. Semin Cell Dev Biol 34: 9-14, 2014.

15. Wang K, Liu F, Zhou LY, Long B, Yuan SM, Wang Y, Liu CY, Sun T, Zhang XJ and Li PF: The long noncoding RNA CHRF regulates cardiac hypertrophy by targeting miR-489. Circ Res 114: 1377-1388, 2014.

16. Shi X, Xu Y, Zhang C, Feng L, Sun Z, Han J, Su F, Zhang Y, Li C and Li X: Subpathway-LNCE: Identify dysfunctional subpathways competitively regulated by lncRNAs through integrating lncRNA-mRNA expression profile and pathway topologies. Oncotarget 7: 69857-69870, 2016.

17. Irizarry RA, Bolstad BM, Collin F, Cope LM, Hobbs B and Speed TP: Summaries of Affymetrix GeneChip probe level data. Nucleic Acids Res 31: e15, 2003.

18. Bolstad BM, Irizarry RA, Astrand M and Speed TP: A comparison of normalization methods for high density oligonucleotide array data based on variance and bias. Bioinformatics 19: 185-193, 2003.
19. Guzzi PH and Cannataro M: Micro-Analyzer: Automatic preprocessing of Affymetrix microarray data. Comput Methods Programs Biomed 111: 402-409, 2013.

20. Allen JD, Wang S, Chen M, Girard L, Minna JD, Xie Y and Xiao G: Probe mapping across multiple microarray platforms. Brief Bioinform 13: 547-554, 2012.

21. Xiao F, Zuo Z, Cai G, Kang S, Gao X and Li T: miRecords: An integrated resource for microRNA-target interactions. Nucleic Acids Res 37 (Database): D105-D110, 2009.

22. Jiang Q, Wang Y, Hao Y, Juan L, Teng M, Zhang X, Li M, Wang $\mathrm{G}$ and Liu Y: miR2Disease: A manually curated database for microRNA deregulation in human disease. Nucleic Acids Res 37 (Database): D98-D104, 2009.

23. Chou CH, Chang NW, Shrestha S, Hsu SD, Lin YL, Lee WH, Yang CD, Hong HC, Wei TY, Tu SJ, et al: miRTarBase 2016: Updates to the experimentally validated miRNA-target interactions database. Nucleic Acids Res 44 (D1): D239-D247, 2016.

24. Vergoulis T, Vlachos IS, Alexiou P, Georgakilas G, Maragkakis M, Reczko M, Gerangelos S, Koziris N, Dalamagas T and Hatzigeorgiou AG: TarBase 6.0: Capturing the exponential growth of miRNA targets with experimental support. Nucleic Acids Res 40 (D1): D222-D229, 2012.

25. Epstein MP, Duncan R, Jiang Y, Conneely KN, Allen AS and Satten GA: A permutation procedure to correct for confounders in case-control studies, including tests of rare variation. Am J Hum Genet 91: 215-223, 2012.

26. Gentilella A, Kozma SC and Thomas G: A liaison between mTOR signaling, ribosome biogenesis and cancer. Biochim Biophys Acta 1849: 812-820, 2015.

27. Iadevaia V, Liu R and Proud CG: mTORC1 signaling controls multiple steps in ribosome biogenesis. Semin Cell Dev Biol 36: 113-120, 2014.

28. Sciarretta S, Volpe M and Sadoshima J: Mammalian target of rapamycin signaling in cardiac physiology and disease. Circ Res 114: 549-564, 2014.

29. Nakielny S, Fischer U, Michael WM and Dreyfuss G: RNA transport. Annu Rev Neurosci 20: 269-301, 1997.

30. Javadov S, Jang S and Agostini B: Crosstalk between mitogen-activated protein kinases and mitochondria in cardiac diseases: Therapeutic perspectives. Pharmacol Ther 144: 202-225, 2014

31. Liao J, He Q, Li M, Chen Y, Liu Y and Wang J: LncRNA MIAT: Myocardial infarction associated and more. Gene 578: 158-161, 2016.

32. Vausort M, Wagner DR and Devaux Y: Long noncoding RNAs in patients with acute myocardial infarction. Circ Res 115: 668-677, 2014.

This work is licensed under a Creative Commons Attribution-NonCommercial-NoDerivatives 4.0 International (CC BY-NC-ND 4.0) License. 